\title{
Patient-reported quality-of-life outcomes in relation to provider-assessed adverse events during head and neck radiotherapy
}

\author{
Joshua R. Niska', Cameron S. Thorpe ${ }^{1}$, Michele Y. Halyard ${ }^{1 *}$ D, Angelina D. Tan², Pamela J. Atherton²,
} Amylou C. Dueck ${ }^{3}$, Samir H. Patel ${ }^{1}$ and Jeff A. Sloan²

\begin{abstract}
Purpose: To assess the relationship between patient-reported quality-of-life (QOL) outcomes and provider-assessed adverse events (AEs) during head-and-neck (H\&N) radiotherapy (RT).

Methods: Sixty-five patients undergoing H\&N RT prospectively completed 12-domain linear analogue selfassessments (LASA) at baseline, before biweekly appointments, and at last week of RT. At the same time points, provider-assessed AEs were graded using Common Terminology Criteria for Adverse Events v4.0. LASA scores were stratified by maximum-grade AE and analyzed using Kruskal-Wallis methodology. Agreement between LASA scores and maximum-grade AE was assessed using Bland-Altman analysis.

Results: Patient-reported QOL outcomes showed clinically meaningful decreases in most domains, predominantly fatigue (77.8\% of patients), social activity (75.4\%), and overall QOL (74.2\%). Provider-assessed AEs showed 100\% grade $2 \mathrm{AE}, 35.4 \%$ grade $3 \mathrm{AE}$, and $3.1 \%$ grade $4 \mathrm{AE}$. At baseline, patients with higher grade AEs reported worse physical well-being (WB) $(P=.04)$. At week 1, the following $\mathrm{QOL}$ domains were worse for patients with higher grade AEs: overall QOL $(P=.03)$, mental WB $(P=.02)$, and physical WB $(P=.03)$. Bland-Altman analysis showed that QOL scores were relatively worse than $A E$ burden at baseline and relatively better at RT completion.

Conclusions: Worse QOL was associated with higher-grade AEs at baseline and early in RT. The impact of AEs on QOL appears to lessen with time. Patient-reported QOL outcomes and provider-assessed AEs provide complementary information.
\end{abstract}

Keywords: Patient reported outcome measures, Quality-of-life, QOL, Radiation oncology, Head and neck neoplasms, Cancer, Radiation therapy, Radiotherapy, RT

\section{Introduction}

Patient-reported outcomes (PROs) can be used to monitor quality-of-life (QOL) in real time during cancer treatment. The use of such data unveils otherwise underappreciated problems, results in improved health-related QOL, and facilitates patient-physician communication, all while not prolonging patient encounters [1-4]. Better patient-reported overall QOL

\footnotetext{
* Correspondence: mhalyard@mayo.edu

'Department of Radiation Oncology, Mayo Clinic, Phoenix, Arizona, USA

Full list of author information is available at the end of the article
}

has been shown to independently predict improved overall survival for a variety of cancers $[5,6]$. Moreover, simply monitoring patient-reported symptoms may improve outcomes. For example, a recent study at Memorial Sloan Kettering Cancer Center, overall survival improved for patients with metastatic cancer who were randomized to longitudinal patient-reported symptom monitoring compared with patients who received usual care (HR, 0.83; $P=.04)$ [7]. Given these demonstrated benefits, clinicians [8], federal agencies [9], and international organizations [10] have increasingly promoted the use of PROs in cancer care. 
Patients with head-and-neck (H\&N) cancer experience well-documented diminished QOL [11-13] and substantial adverse events (AEs) during radiotherapy (RT), with or without chemotherapy $[11,13,14]$. It may seem obvious that QOL should decline with increasingly severe AEs. However, the relationship between patient-reported QOL and AEs is poorly defined. In a pooled analysis of 6 lung cancer trials, patient-reported QOL and patientreported $\mathrm{AEs}$ had moderate agreement [15]. In a similar pooled analysis of 12 lung cancer trials, patient-reported QOL and provider-assessed AEs had low agreement [16]. To our knowledge, the relationship between patientreported QOL and AEs has not yet been evaluated in $\mathrm{H} \& \mathrm{~N}$ cancer. Given the acute challenges patients face during RT for $\mathrm{H} \& \mathrm{~N}$ cancer, understanding QOL and AEs over the course of their treatment is of interest.

Real-time, web-based electronic PROs (ePROs) have proven feasible in outpatient medical oncology [17] and radiation oncology [11] settings. RT provides a unique opportunity to monitor multiple time points during treatment, given that patients are treated daily for several weeks and assessed by providers at least weekly. Our group has previously used ePROs to characterize QOL changes and the burden of AEs at multiple time points during H\&N RT [11]. While our prior work independently characterized patient-reported QOL and provider-assessed AEs, we perceived a link between the 2 end points. We hypothesized that diminished patientreported QOL would be associated with increasingly severe provider-assessed AEs during H\&N RT. To address this question, we compared 12 patient-reported QOL domains with provider-assessed AE severity across 5 time points during RT for the subset of 65 patients with $\mathrm{H} \& \mathrm{~N}$ cancer enrolled in a previously completed prospective trial approved by the institutional review board.

\section{Methods and materials}

Study design, study measures, prospective data collection, and retrospective data collection have been previously described [11]. Briefly, patients from the previous prospective trial were included in this study if they had undergone curative-intent RT for nonmetastatic $H \& N$ cancer. At baseline, before biweekly appointments, and at last week of RT, patients prospectively completed electronic, real-time linear analogue self-assessments (LASA) to assess 12 domains: overall QOL, mental wellbeing (WB), physical WB, emotional WB, social activity, spiritual WB, pain frequency, pain severity, fatigue level, level of support, financial concerns, and legal concerns $[18,19]$. Patient, disease, and treatment characteristics were retrospectively collected from electronic health records. Provider-assessed AEs were retrospectively collected for the same time points as the prospective patient-reported LASA data and graded using Common
Terminology Criteria for Adverse Events (CTCAE). CTCAE Version 4.0 includes 790 distinct adverse events that are not necessarily symptom-based (National Cancer Institute, Version 4.0, May 28, 2009).

Scores in each LASA domain were transformed to a 0to 100 - point scale, with 0 being worst and 100 being best. Changes between time points were calculated. A change of 10 or more points on the 0 - to 100-point scale was considered clinically meaningful [6]. At each time point, patients were categorized by maximum-grade $\mathrm{AE}$. To match the 0- to 100-point LASA scale, AE grade was transformed to a 0 - to 100 -point scale (grade $0=100$, $1=80,2=60,3=40,4=20$, and $5=0$ ). LASA scores were compared to maximum-grade AE using KruskalWallis methodology. Bland-Altman analysis was used to assess agreement between LASA scores and maximumgrade AE [15] Spearman correlation coefficients were used to assess the size of correlations using the criteria published by Cohen: low correlation, 0.10 to 0.29 ; moderate correlation, 0.30 to 0.49 ; and high correlation, > 0.50 [20]. All hypothesis testing was completed using 2sided alternative hypothesis and 5\% type I error.

\section{Results}

Table 1 presents patient, disease, and treatment characteristics for the 65 patients who met the inclusion criteria. Figure 1 displays mean LASA scores across the 12 LASA domains at each time point over the course of RT [11]. Most patients reported clinically meaningful decreases ( $\geq 10$ points on the $0-100$ scale) at some point during RT in the following QOL domains: overall QOL, mental WB, physical WB, emotional WB, social activity, spiritual WB, pain frequency, pain severity, and fatigue level. The QOL domains with the most widespread, clinically meaningful decrease were fatigue $(77.8 \%$ of patients), social activity (75.4\%), and overall QOL (74.2\%). At end of RT (week 7), mean scores in the following QOL domains were worse than baseline: overall QOL, mental WB, physical WB, emotional WB, social activity, pain frequency, pain severity, and fatigue level. A more detailed description of the ePROs for QOL has been previously published [11].

Figure 2 shows the distribution of maximum-grade AEs at each time point during RT. The area of shading for a given $\mathrm{AE}$ grade is proportional to the number of patients with that maximum-grade $\mathrm{AE}$ at that time point. The corresponding raw number of patients experiencing a given maximum-grade $\mathrm{AE}$ at each time point is also shown. For reference, at week 3 , all patients had an $\mathrm{AE} ; 16$ patients had maximum grade $1 \mathrm{AE} ; 41$ patients, maximum grade $2 \mathrm{AE} ; 6$ patients, maximum grade $3 \mathrm{AE}$; and 2 patients, maximum grade $4 \mathrm{AE}$. Every patient experienced at least grade $2 \mathrm{AE}$ during $\mathrm{RT}$, $35.4 \%$ at least grade 3 , and $3.1 \%$ grade 4 . All but 1 
Table 1 Patient, disease, and treatment characteristics $(N=65)$

\begin{tabular}{|c|c|c|c|c|c|}
\hline Age & Mean (SD) & $65.3(12.6)$ & & & No. (\%) \\
\hline & & & Staging & Stage I & $6(9.2)$ \\
\hline \multirow[t]{3}{*}{ Radiation Dose } & Median (Range) in Gy & $60(50-70)$ & & Stage II & $9(13.8)$ \\
\hline & & & & Stage III & $10(15.4)$ \\
\hline & & No. (\%) & & Stage IV & $39(60.0)$ \\
\hline \multirow[t]{3}{*}{ Sex } & Male & $52(80.0)$ & & Recurrent & $12(18.5)$ \\
\hline & Female & $13(20.0)$ & & & \\
\hline & & & Primary Site & Oral cavity/oropharynx & $28(43.1)$ \\
\hline \multirow[t]{6}{*}{ Race } & White & $60(92.3)$ & & Larynx & $8(12.3)$ \\
\hline & Black & $2(3.1)$ & & Salivary gland & $7(10.8)$ \\
\hline & Asian & $1(1.5)$ & & Skin & $6(9.2)$ \\
\hline & Pacific Islander & $1(1.5)$ & & Nasopharynx & $5(7.7)$ \\
\hline & $\mathrm{Al} / \mathrm{AN}$ & $1(1.5)$ & & Nasal cavity/paranasal sinus & $4(6.2)$ \\
\hline & & & & Other & $7(10.8)$ \\
\hline \multirow[t]{5}{*}{ Treatment Type } & Adjuvant CCRT & $21(32.3)$ & & & \\
\hline & Adjuvant RT & $20(30.8)$ & Histology & Squamous cell carcinoma & $51(78.5)$ \\
\hline & Definitive CCRT & $19(29.2)$ & & Spindle cell carcinoma & $2(3.1)$ \\
\hline & Definitive RT & $5(7.7)$ & & Papillary thyroid carcinoma & $2(3.1)$ \\
\hline & & & & Melanoma & $2(3.1)$ \\
\hline \multirow[t]{4}{*}{ Chemotherapy } & Cisplatin & $25(38.5)$ & & Merkel cell carcinoma & $2(3.1)$ \\
\hline & Cetuximab & $8(12.3)$ & & Adenocarcinoma & $2(3.1)$ \\
\hline & Carboplatin & $5(7.7)$ & & Other & $4(6.2)$ \\
\hline & Other & $2(3.1)$ & & & \\
\hline
\end{tabular}

Abbreviations: Al/AN, American Indian/Alaska Native; CCRT, concurrent chemoradiotherapy; RT, radiotherapy Note: Race was self-identified. Percentages do not add to 100 because of rounding

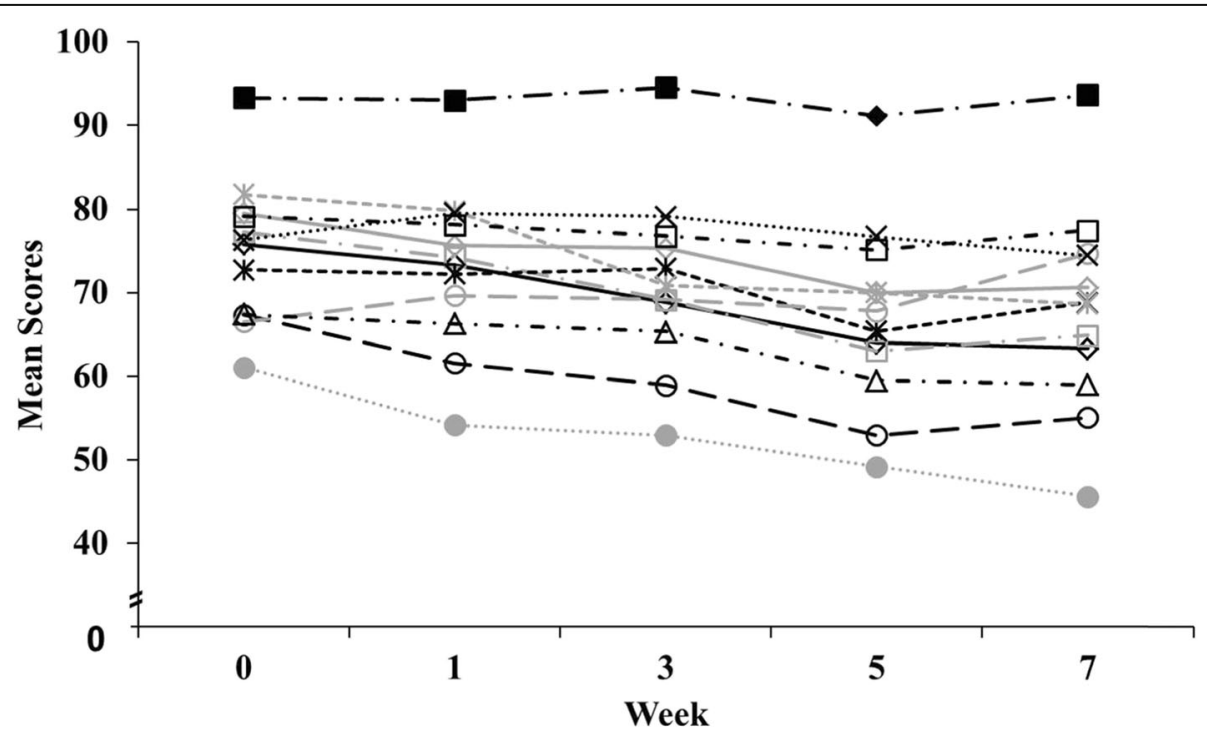

\begin{tabular}{|c|c|c|c|}
\hline$\diamond$ Overall QOL & $\smile$ Mental WB & $\square$ Pain Frequency & -*-Emotional WB \\
\hline$\multimap$-Social Activity & -*-Pain Severity & $\smile$-Financial Concerns & $-\Delta-$ Physical WB \\
\hline .. - Fatigue & $-\bullet-$ Spiritual WB & ..× $\times$ Legal Concerns & Level of Support \\
\hline
\end{tabular}

Fig. 1 Average LASA scores from baseline to RT completion $(0=$ Low QOL; $100=$ High QOL). Abbreviations: LASA, linear analog self-assessment; QOL, quality of life; WB, well-being. Previously published data [Niska 2017] 


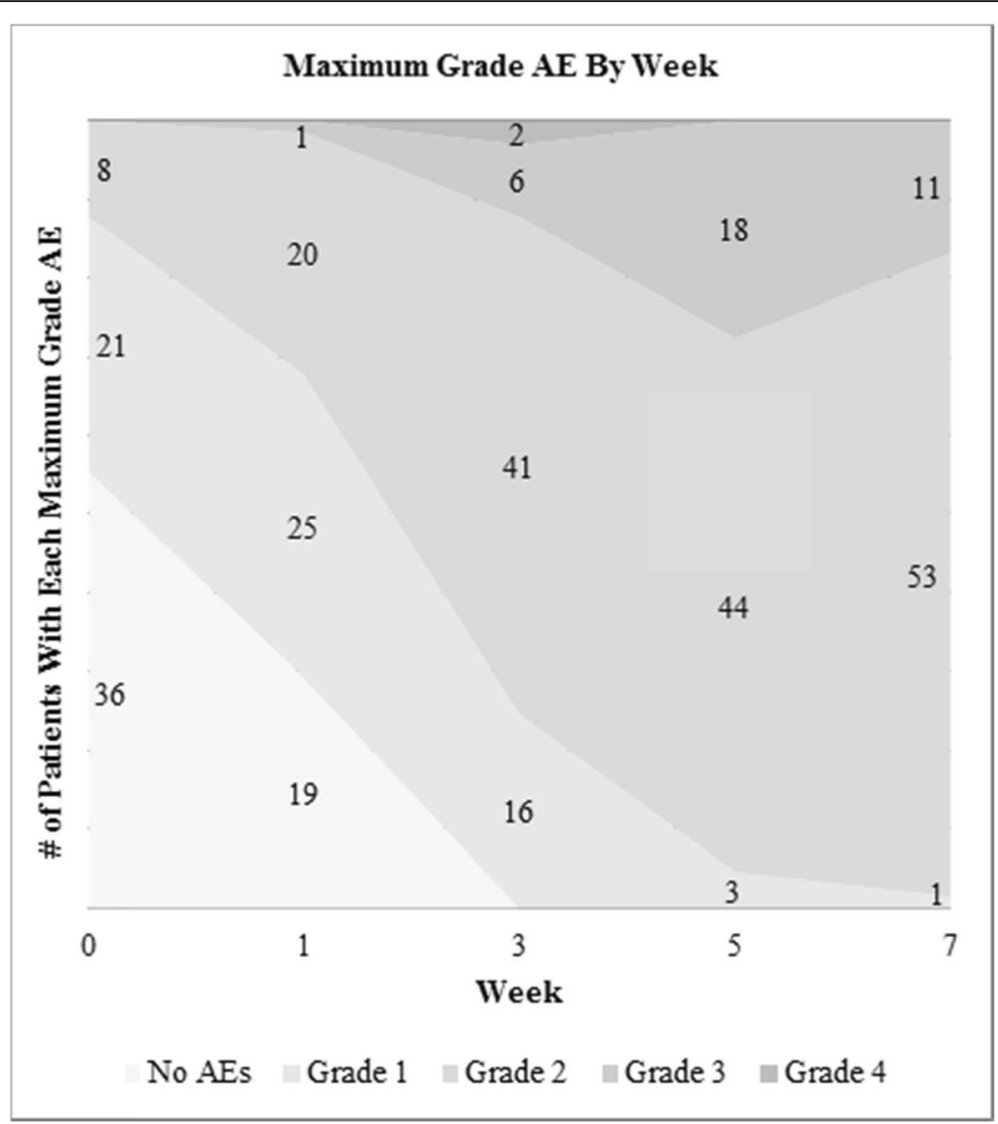

Fig. 2 Distribution of maximum grade AE by week. For reference, maximum grade AE distribution at week 3 was grade 1: 16 patients; grade 2: 41 patients; grade 3: 6 patients; grade 4: 2 patients

patient had at least a grade $2 \mathrm{AE}$ at $\mathrm{RT}$ completion. The incidence of specific AEs and their grades over the course of RT have been previously published [11].

For subsequent analyses, the only patient with a grade 3 $\mathrm{AE}$ at week 1 was excluded as an outlier, and the 2 patients with grade 4 AEs at week 3 were excluded due to missing data. Table 2 shows the week-by-week mean LASA score for each of the 12 QOL domains. QOL scores are separated into 4 groups: patients experiencing no $\mathrm{AE}$ (grade 0 ), patients experiencing maximum grade $1 \mathrm{AE}$, patients experiencing maximum grade $2 \mathrm{AE}$, and patients experiencing maximum grade $3 \mathrm{AE}$. $P$ values are provided to indicate whether patient-reported QOL was significantly different for patients experiencing different grades of AEs. Over the course of RT, multiple QOL domains followed the same general trend regarding maximum grade AE: over the first 3 time points, patients with only grade 1 AEs reported better mean QOL scores, while over the last 2 time points this trend was no longer evident. This pattern was consistent across overall QOL, mental WB, physical WB, emotional WB, social activity, spiritual WB, pain frequency, pain severity, fatigue, and level of support. The only statistically significant QOL differences by maximum-grade $\mathrm{AE}$ occurred at baseline (physical WB) and week 1 (overall QOL, mental WB, physical WB). Of note, each domain that was statistically significant (bold in Table 2) also averaged a clinically meaningful change in LASA score ( $\geq 10$ points on the $0-100$ scale). In weeks 3, 5, and 7, QOL scores showed no clear relation to maximum-grade $\mathrm{AE}$.

Where QOL score was statistically different by maximum-grade AE, Spearman correlation coefficients were 0.34 for week 1 overall QOL (moderate correlation), 0.35 for week 1 mental WB (moderate), 0.18 for baseline physical WB (low), and 0.28 for week 1 physical WB (low). Bland-Altman analysis, used to assess agreement between AE grade and LASA score at each time point, revealed differential agreement over the course of RT (presented as mean LASA score minus mean AE score at each time point, Fig. 3). After transforming to 0 to 100 scale, mean AE score minus mean LASA score was lower at baseline than week 7 across all QOL domains.

\section{Discussion}

To our knowledge, our study provides the first assessment of the relationship between patient-reported QOL 
Table 2 QOL Scores by Maximum Grade Adverse Event Per Week

\begin{tabular}{|c|c|c|c|c|c|c|c|c|c|c|c|c|c|}
\hline & & Grade 0 & Grade 1 & Grade 2 & Grade 3 & & & & Grade 0 & Grade 1 & Grade 2 & Grade 3 & \\
\hline & Week & $\begin{array}{l}\text { Mean } \\
\text { (SD) }\end{array}$ & $\begin{array}{l}\text { Mean } \\
\text { (SD) }\end{array}$ & $\begin{array}{l}\text { Mean } \\
\text { (SD) }\end{array}$ & $\begin{array}{l}\text { Mean } \\
\text { (SD) }\end{array}$ & $P$-value & & Week & $\begin{array}{l}\text { Mean } \\
\text { (SD) }\end{array}$ & $\begin{array}{l}\text { Mean } \\
\text { (SD) }\end{array}$ & $\begin{array}{l}\text { Mean } \\
\text { (SD) }\end{array}$ & $\begin{array}{l}\text { Mean } \\
\text { (SD) }\end{array}$ & $P$ P-value \\
\hline \multirow[t]{5}{*}{ Overall QOL } & 0 & $\begin{array}{l}78.8 \\
(20.0)\end{array}$ & $\begin{array}{l}75.7 \\
(16.3)\end{array}$ & $\begin{array}{l}61.4 \\
(24.1)\end{array}$ & & 0.15 & Pain Frequency & 0 & $\begin{array}{l}84.0 \\
(18.7)\end{array}$ & $\begin{array}{l}74.3 \\
(23.8)\end{array}$ & $\begin{array}{l}52.9 \\
(39.9)\end{array}$ & & 0.09 \\
\hline & 1 & $\begin{array}{l}81.7 \\
(20.4)\end{array}$ & $\begin{array}{l}74.6 \\
(24.1)\end{array}$ & $\begin{array}{l}64.5 \\
(20.1)\end{array}$ & & 0.03 & & 1 & $\begin{array}{l}72.4 \\
(34.0)\end{array}$ & $\begin{array}{l}75.8 \\
(28.3)\end{array}$ & $\begin{array}{l}74.0 \\
(23.5)\end{array}$ & & 0.81 \\
\hline & 3 & & $\begin{array}{l}79.3 \\
(16.7)\end{array}$ & $\begin{array}{l}64.7 \\
(21.4)\end{array}$ & $\begin{array}{l}68.0 \\
(32.7)\end{array}$ & 0.09 & & 3 & & $\begin{array}{l}78.0 \\
(21.1)\end{array}$ & $\begin{array}{l}65.0 \\
(23.0)\end{array}$ & $\begin{array}{l}74.0 \\
(20.7)\end{array}$ & 0.15 \\
\hline & 5 & & $\begin{array}{l}63.3 \\
(15.3)\end{array}$ & $\begin{array}{l}65.2 \\
(21.4)\end{array}$ & $\begin{array}{l}60.7 \\
(22.8)\end{array}$ & 0.72 & & 5 & & $\begin{array}{l}50.0 \\
(28.3)\end{array}$ & $\begin{array}{l}64.1 \\
(24.2)\end{array}$ & $\begin{array}{l}61.9 \\
(25.9)\end{array}$ & 0.69 \\
\hline & 7 & & & $\begin{array}{l}62.1 \\
(20.0)\end{array}$ & $\begin{array}{l}77.5 \\
(20.6)\end{array}$ & 0.16 & & 7 & & & $\begin{array}{l}65.7 \\
(26.5)\end{array}$ & $\begin{array}{l}55.0 \\
(26.5)\end{array}$ & 0.35 \\
\hline \multirow[t]{5}{*}{ Mental WB } & 0 & $\begin{array}{l}80.6 \\
(20.3)\end{array}$ & $\begin{array}{l}80.5 \\
(14.7)\end{array}$ & $\begin{array}{l}71.4 \\
(21.2)\end{array}$ & & 0.43 & Pain Severity & 0 & $\begin{array}{l}86.3 \\
(15.0)\end{array}$ & $\begin{array}{l}78.6 \\
(17.7)\end{array}$ & $\begin{array}{l}68.6 \\
(34.4)\end{array}$ & & 0.22 \\
\hline & 1 & $\begin{array}{l}81.7 \\
(20.9)\end{array}$ & $\begin{array}{l}79.2 \\
(17.4)\end{array}$ & $\begin{array}{l}66.0 \\
(19.0)\end{array}$ & & 0.02 & & 1 & $\begin{array}{l}86.3 \\
(17.5)\end{array}$ & $\begin{array}{l}77.9 \\
(24.8)\end{array}$ & $\begin{array}{l}77.0 \\
(22.0)\end{array}$ & & 0.32 \\
\hline & 3 & & $\begin{array}{l}78.7 \\
(16.4)\end{array}$ & $\begin{array}{l}73.7 \\
(18.9)\end{array}$ & $\begin{array}{l}78.0 \\
(29.5)\end{array}$ & 0.49 & & 3 & & $\begin{array}{l}80.0 \\
(15.1)\end{array}$ & $\begin{array}{l}67.9 \\
(21.3)\end{array}$ & $\begin{array}{l}66.0 \\
(28.8)\end{array}$ & 0.20 \\
\hline & 5 & & $63.3(5.8)$ & $\begin{array}{l}71.2 \\
(22.0)\end{array}$ & $\begin{array}{l}68.1 \\
(23.4)\end{array}$ & 0.57 & & 5 & & $\begin{array}{l}60.0 \\
(28.3)\end{array}$ & $\begin{array}{l}71.0 \\
(21.0)\end{array}$ & $\begin{array}{l}68.8 \\
(20.9)\end{array}$ & 0.67 \\
\hline & 7 & & & $\begin{array}{l}69.4 \\
(18.8)\end{array}$ & $\begin{array}{l}85.0 \\
(12.9)\end{array}$ & 0.10 & & 7 & & & $\begin{array}{l}69.4 \\
(23.2)\end{array}$ & $\begin{array}{l}60.0 \\
(18.3)\end{array}$ & 0.34 \\
\hline \multirow[t]{5}{*}{ Physical WB } & 0 & $\begin{array}{l}69.4 \\
(19.1)\end{array}$ & $\begin{array}{l}70.5 \\
(17.2)\end{array}$ & $\begin{array}{l}48.6 \\
(19.5)\end{array}$ & & 0.04 & Fatigue & 0 & $\begin{array}{l}66.0 \\
(27.1)\end{array}$ & $\begin{array}{l}55.7 \\
(26.9)\end{array}$ & $\begin{array}{l}52.9 \\
(27.5)\end{array}$ & & 0.22 \\
\hline & 1 & $\begin{array}{l}71.1 \\
(24.7)\end{array}$ & $\begin{array}{l}71.3 \\
(23.3)\end{array}$ & $\begin{array}{l}56.0 \\
(17.9)\end{array}$ & & 0.03 & & 1 & $\begin{array}{l}60.6 \\
(25.3)\end{array}$ & $\begin{array}{l}59.2 \\
(26.2)\end{array}$ & $\begin{array}{l}42.5 \\
(17.4)\end{array}$ & & 0.08 \\
\hline & 3 & & $\begin{array}{l}70.7 \\
(17.5)\end{array}$ & $\begin{array}{l}63.7 \\
(20.5)\end{array}$ & $\begin{array}{l}62.0 \\
(25.9)\end{array}$ & 0.58 & & 3 & & $\begin{array}{l}57.3 \\
(27.9)\end{array}$ & $\begin{array}{l}50.3 \\
(25.8)\end{array}$ & $\begin{array}{l}60.0 \\
(15.8)\end{array}$ & 0.50 \\
\hline & 5 & & $\begin{array}{l}60.0 \\
(20.0)\end{array}$ & $\begin{array}{l}61.5 \\
(21.9)\end{array}$ & $\begin{array}{l}54.4 \\
(25.8)\end{array}$ & 0.62 & & 5 & & $\begin{array}{l}33.3 \\
(11.5)\end{array}$ & $\begin{array}{l}49.8 \\
(24.2)\end{array}$ & $\begin{array}{l}50.6 \\
(18.4)\end{array}$ & 0.36 \\
\hline & 7 & & & $\begin{array}{l}57.9 \\
(19.7)\end{array}$ & $\begin{array}{l}72.5 \\
(17.1)\end{array}$ & 0.16 & & 7 & & & $\begin{array}{l}44.7 \\
(23.3)\end{array}$ & $\begin{array}{l}60.0 \\
(34.6)\end{array}$ & 0.48 \\
\hline \multirow[t]{5}{*}{$\begin{array}{l}\text { Emotional } \\
\text { WB }\end{array}$} & 0 & $\begin{array}{l}75.6 \\
(20.8)\end{array}$ & $\begin{array}{l}72.9 \\
(17.1)\end{array}$ & $\begin{array}{l}58.6 \\
(23.4)\end{array}$ & & 0.15 & $\begin{array}{l}\text { Level of } \\
\text { Support }\end{array}$ & 0 & $\begin{array}{l}94.3 \\
(11.4)\end{array}$ & $\begin{array}{l}94.3 \\
(12.1)\end{array}$ & $\begin{array}{l}85.7 \\
(29.9)\end{array}$ & & 0.94 \\
\hline & 1 & $\begin{array}{l}77.2 \\
(22.7)\end{array}$ & $\begin{array}{l}72.9 \\
(21.8)\end{array}$ & $\begin{array}{l}67.0 \\
(20.3)\end{array}$ & & 0.22 & & 1 & $\begin{array}{l}93.9 \\
(16.1)\end{array}$ & $\begin{array}{l}94.6 \\
(7.8)\end{array}$ & $\begin{array}{l}90.5 \\
(15.4)\end{array}$ & & 0.31 \\
\hline & 3 & & $\begin{array}{l}78.7 \\
(13.0)\end{array}$ & $\begin{array}{l}70.3 \\
(22.1)\end{array}$ & $\begin{array}{l}76.0 \\
(33.6)\end{array}$ & 0.44 & & 3 & & $\begin{array}{l}95.0 \\
(8.5)\end{array}$ & $\begin{array}{l}94.5 \\
(10.3)\end{array}$ & $\begin{array}{l}94.0 \\
(13.4)\end{array}$ & 0.93 \\
\hline & 5 & & $\begin{array}{l}66.7 \\
(11.5)\end{array}$ & $\begin{array}{l}65.0 \\
(21.6)\end{array}$ & $\begin{array}{l}66.3 \\
(24.7)\end{array}$ & 0.94 & & 5 & & $\begin{array}{l}100.0 \\
(0.0)\end{array}$ & $\begin{array}{l}91.2 \\
(15.0)\end{array}$ & $\begin{array}{l}89.4 \\
(15.3)\end{array}$ & 0.30 \\
\hline & 7 & & & $\begin{array}{l}68.1 \\
(19.9)\end{array}$ & $\begin{array}{l}77.5 \\
(20.6)\end{array}$ & 0.34 & & 7 & & & $\begin{array}{l}93.5 \\
(11.4)\end{array}$ & $\begin{array}{l}96.7 \\
(5.8)\end{array}$ & 0.74 \\
\hline \multirow[t]{5}{*}{$\begin{array}{l}\text { Social } \\
\text { Activity }\end{array}$} & 0 & $\begin{array}{l}71.2 \\
(25.9)\end{array}$ & $\begin{array}{l}67.5 \\
(20.5)\end{array}$ & $\begin{array}{l}48.6 \\
(38.0)\end{array}$ & & 0.23 & $\begin{array}{l}\text { Financial } \\
\text { Concerns }\end{array}$ & 0 & $\begin{array}{l}64.9 \\
(31.9)\end{array}$ & $\begin{array}{l}63.8 \\
(35.1)\end{array}$ & $\begin{array}{l}82.9 \\
(37.3)\end{array}$ & & 0.21 \\
\hline & 1 & $\begin{array}{l}63.9 \\
(31.5)\end{array}$ & $\begin{array}{l}65.0 \\
(27.0)\end{array}$ & $\begin{array}{l}55.5 \\
(21.9)\end{array}$ & & 0.41 & & 1 & $\begin{array}{l}67.2 \\
(33.7)\end{array}$ & $\begin{array}{l}66.7 \\
(30.3)\end{array}$ & $\begin{array}{l}75.5 \\
(26.8)\end{array}$ & & 0.68 \\
\hline & 3 & & $\begin{array}{l}71.3 \\
(21.0)\end{array}$ & $\begin{array}{l}54.5 \\
(25.9)\end{array}$ & $\begin{array}{l}56.0 \\
(31.3)\end{array}$ & 0.11 & & 3 & & $\begin{array}{l}64.7 \\
(34.2)\end{array}$ & $\begin{array}{l}70.0 \\
(31.5)\end{array}$ & $\begin{array}{l}76.0 \\
(37.8)\end{array}$ & 0.69 \\
\hline & 5 & & $\begin{array}{l}66.7 \\
(25.2)\end{array}$ & $\begin{array}{l}54.5 \\
(25.9)\end{array}$ & $\begin{array}{l}46.3 \\
(27.5)\end{array}$ & 0.40 & & 5 & & $\begin{array}{l}93.3 \\
(11.5)\end{array}$ & $\begin{array}{l}69.8 \\
(32.2)\end{array}$ & $\begin{array}{l}58.1 \\
(32.3)\end{array}$ & 0.10 \\
\hline & 7 & & & $\begin{array}{l}54.5 \\
(23.0)\end{array}$ & $\begin{array}{l}62.5 \\
(33.0)\end{array}$ & 0.51 & & 7 & & & $\begin{array}{l}75.7 \\
(26.1)\end{array}$ & $\begin{array}{l}60.0 \\
(52.9)\end{array}$ & 0.72 \\
\hline Spiritual WB & 0 & $\begin{array}{l}81.5 \\
(20.9)\end{array}$ & $\begin{array}{l}78.0 \\
(17.9)\end{array}$ & $\begin{array}{l}70.0 \\
(26.8)\end{array}$ & & 0.42 & Legal Concerns & 0 & $\begin{array}{l}74.6 \\
(34.0)\end{array}$ & $\begin{array}{l}72.4 \\
(33.2)\end{array}$ & $\begin{array}{l}97.1 \\
(7.6)\end{array}$ & & 0.07 \\
\hline
\end{tabular}


Table 2 QOL Scores by Maximum Grade Adverse Event Per Week (Continued)

\begin{tabular}{|c|c|c|c|c|c|c|c|c|c|c|c|}
\hline & Grade 0 & Grade 1 & Grade 2 & Grade 3 & & & Grade 0 & Grade 1 & Grade 2 & Grade 3 & \\
\hline 1 & $\begin{array}{l}82.8 \\
(17.4)\end{array}$ & $\begin{array}{l}77.1 \\
(25.6)\end{array}$ & $\begin{array}{l}74.7 \\
(25.0)\end{array}$ & & 0.82 & 1 & $\begin{array}{l}84.4 \\
(25.0)\end{array}$ & $\begin{array}{l}77.1 \\
(35.1)\end{array}$ & $\begin{array}{l}78.0 \\
(32.5)\end{array}$ & & 0.77 \\
\hline 3 & & $\begin{array}{l}78.0 \\
(17.4)\end{array}$ & $\begin{array}{l}76.5 \\
(20.7)\end{array}$ & $\begin{array}{l}75.0 \\
(37.9)\end{array}$ & 0.89 & 3 & & $\begin{array}{l}66.0 \\
(37.0)\end{array}$ & $\begin{array}{l}82.9 \\
(28.2)\end{array}$ & $\begin{array}{l}90.0 \\
(22.4)\end{array}$ & 0.07 \\
\hline 5 & & $\begin{array}{l}90.0 \\
(10.0)\end{array}$ & $\begin{array}{l}75.3 \\
(21.7)\end{array}$ & $\begin{array}{l}72.0 \\
(26.8)\end{array}$ & 0.49 & 5 & & $\begin{array}{l}86.7 \\
(23.1)\end{array}$ & $\begin{array}{l}78.8 \\
(31.3)\end{array}$ & $\begin{array}{l}69.4 \\
(34.7)\end{array}$ & 0.52 \\
\hline 7 & & & $\begin{array}{l}76.7 \\
(20.9)\end{array}$ & $\begin{array}{l}85.0 \\
(19.1)\end{array}$ & 0.40 & 7 & & & $\begin{array}{l}76.9 \\
(32.1)\end{array}$ & $\begin{array}{l}47.5 \\
(37.7)\end{array}$ & 0.15 \\
\hline
\end{tabular}

Analyzed using Kruskal-Wallis methodology

The only patient with grade 3 adverse event at week 1 was excluded

The only two patients with grade 4 adverse events were excluded

and provider-assessed AEs during H\&N RT. Although H\&N RT can impact all aspects of QOL, fatigue, social activity, and overall QOL are most commonly affected. All patients experienced grade $2 \mathrm{AE}$, many experienced grade $3 \mathrm{AE}$, and few experienced grade $4 \mathrm{AE}$. Excluding 1 outlier, grade 3 AEs began in week 3 and peaked before end of RT. At baseline and early in RT, clinically meaningful decreases in QOL were associated with more severe AEs, with low to moderate correlation. As time passed, QOL lost its correlation with AE severity.

Although QOL worsened and AE severity increased over the course of RT, correlation between them was only present at baseline and week 1 . Toxicities at these early time points are more likely to be caused by prior treatment (eg, surgery or chemotherapy) or by the disease itself, and less likely to be caused by RT. Patient anticipation of upcoming RT may also play a role. Of note, the toxicities that correlated with clinically meaningful decreases in QOL were all grade 2. Understandably, clinicians often focus on grade 3 or higher AEs late in RT or after RT completion. Nonetheless, early grade 2 AEs appear to be negatively associated with patient-reported QOL and should be proactively addressed.

As any clinician can attest, patient concerns and provider concerns are often incongruent. For example, in a phase II trial of metastatic prostate cancer, physician-reported symptoms were only $47 \%$ sensitive and $68 \%$ specific for patient-reported symptoms [21]. Prior studies in lung cancer have shown low correlation between patient-reported QOL and provider-assessed AEs [16] but moderate correlation between patientreported QOL and patient-reported AEs [15]. Furthermore, agreement between provider-assessed AEs and patient-reported AEs has been shown to decline

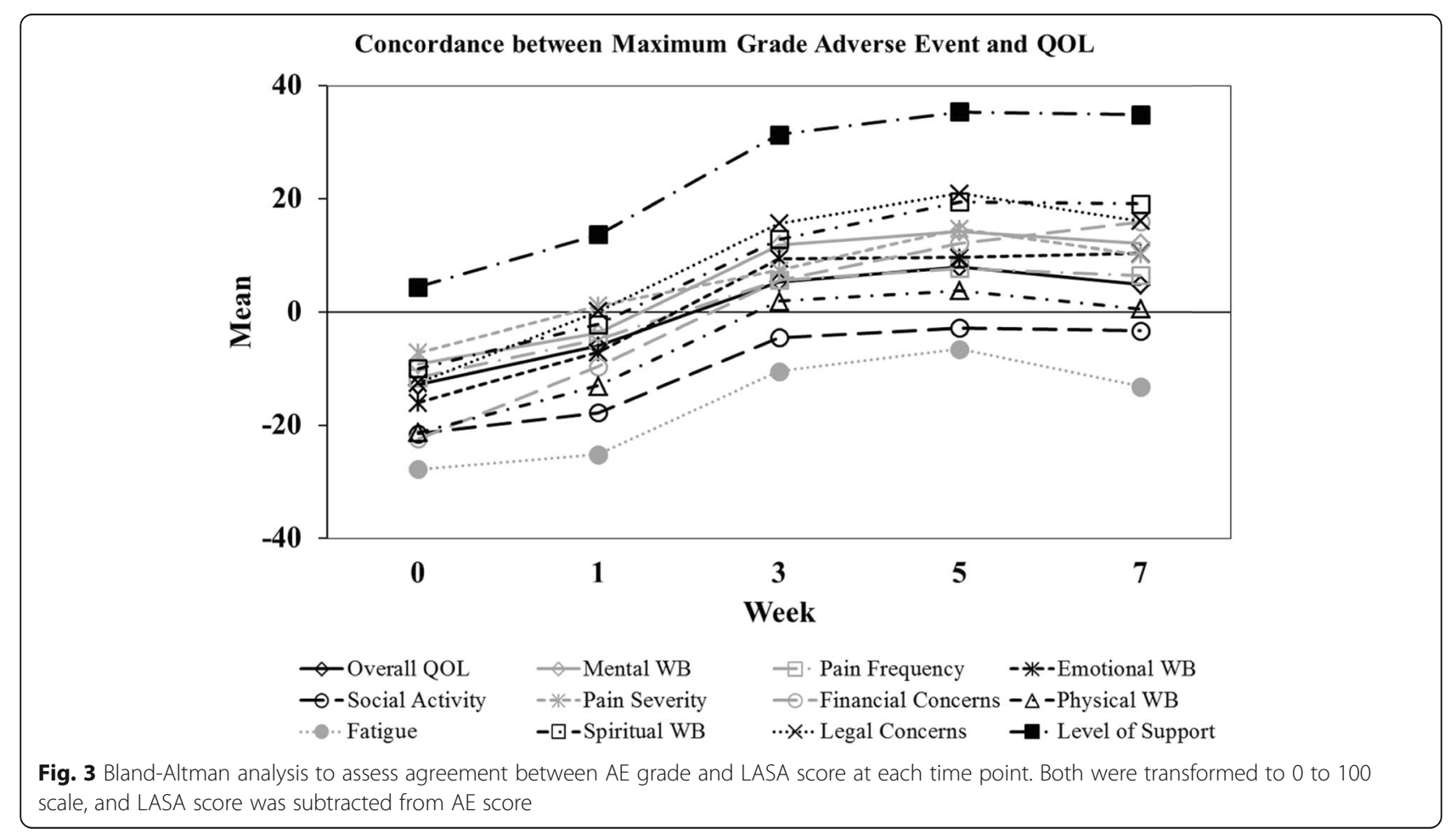


throughout H\&N RT [22]. To our knowledge, there are no reports in the literature comparing patientreported QOL and provider-assessed AEs for patients with $\mathrm{H} \& \mathrm{~N}$ cancer. We showed low to moderate correlation between patient-reported QOL and providerassessed AEs at early time points for patients having H\&N RT. No correlation was present at later time points. This rapid decline in correlation between patient-reported QOL and provider-assessed AEs may be due to a number of factors: patients may become accustomed to mounting treatment-related toxicities, providers may be adequately addressing patient concerns, or perhaps QOL becomes truly independent of AEs as patients become more resilient.

Our study has several limitations. This was an unplanned secondary analysis of data from a prospective clinical trial. As such, our findings should be treated as hypothesis-generating and need to be confirmed by future prospective studies. The demographics of our institution may limit the generalizability of our results $(92.3 \%$ of patients included in the study were white). With only 65 patients, statistical power was limited. Two patients with maximum grade $4 \mathrm{AE}$ at week 3 were excluded due to missing data. Furthermore, to minimize the effect of a single outlier on our analysis, we excluded the 1 patient with maximum grade $3 \mathrm{AE}$ at week 1 . We had relatively few AEs $\geq$ grade 3 . However, early grade 2 AEs did predict QOL decrement and may portend future grade 3 AEs. In a larger sample with more high-grade AEs, we may indeed find association between $\geq$ grade 3 AEs and QOL. Furthermore, using a QOL instrument specific to $H \& N$ cancer may show greater association with AEs. Additionally, we only captured AEs and QOL during RT. In the pooled analysis of lung cancer trials by Huschka et al., moderate agreement between $\geq$ grade 3 AEs and QOL was seen after RT [15]. In the future, capturing data both during and after RT may allow for better characterization of the relationship between patient-reported QOL and provider-assessed AEs. Moreover, our study did not include patientreported AEs, which are emerging as an important complementary end point for future trials [23].

\section{Conclusions}

During H\&N RT, patient-reported QOL has a complex relationship with provider-assessed AEs. At least initially, grade 2 AEs matter to patients: clinically meaningful decreases in overall QOL, mental WB, and physical WB have low to moderate correlation with grade 2 AEs at baseline and early in RT. Over the course of RT, QOL worsens, and the burden of AEs increases. However, patient-reported QOL does not correlate with provider-assessed AEs after week 1 . Therefore, patient-reported QOL complements provider-assessed AEs during $\mathrm{H} \& \mathrm{~N}$ RT. The role of $p a-$ tient-reported AEs continues to evolve. Going forward, clinical trials that include patient-reported QOL, patient-reported AEs, and provider-assessed AEs may ultimately improve cancer care.

\section{Abbreviations}

AE: Adverse event; CTCAE: Common terminology criteria for adverse events; ePROs: Electronic patient-reported outcomes; H\&N: Head and neck; LASA: Linear analog self-assessment; PROs: Patient-reported outcomes; QOL: Quality-of-life; RT: Radiotherapy; WB: Well-being

\section{Acknowledgements}

This research was not supported by grant or other financial support. This data has previously been presented in abstract form at the following meetings: 2016 Multidisciplinary Head and Neck Cancer Symposium, 2016 ASTRO Annual Meeting, and the 2016 International Society for Quality of Life Research Annual Conference.

\section{Authors' contributions}

JRN analyzed and interpreted the data and was a major contributor in writing and revising the draft. CST was a major contributor in interpreting the data, writing and revising the draft. MYH was critical to the conception, design, acquisition of data, and interpretation of the study. MYH also provided substantial revisions. ADT and PJA were both substantially involved in the analysis of the data. ACD was a major contributor in the analysis of the data and provided substantial revisions. SHP contributed to the design of the study, acquisition of the data, and to revising the manuscript. JAS contributed to the design and analysis of the work, and contributed to revising the manuscript. All authors read and approved the final manuscript.

\section{Funding}

There is no funding for this research.

\section{Availability of data and materials}

We have full control over the primary data and will allow it to be reviewed if requested.

Ethics approval and consent to participate

All patients provided informed consent to participate in the study. The study was approved by the Mayo Clinic Institutional Review Board.

\section{Consent for publication}

Not applicable.

\section{Competing interests}

Actual or potential conflicts of interest do not exist.

\section{Author details}

'Department of Radiation Oncology, Mayo Clinic, Phoenix, Arizona, USA. ${ }^{2}$ Division of Biomedical Statistics and Informatics, Mayo Clinic, Rochester, Minnesota, USA. ${ }^{3}$ Department of Health Sciences Research, Mayo Clinic, Scottsdale, Arizona, USA.

Received: 18 December 2019 Accepted: 5 July 2020

Published online: 16 July 2020

\section{References}

1. Berry, D. L., Blumenstein, B. A., Halpenny, B., Wolpin, S., Fann, J. R., AustinSeymour, M., ... McCorkle, R. (2011). Enhancing patient-provider communication with the electronic self-report assessment for cancer: A randomized trial. J Clin Oncol, 29(8), 1029-1035. https://doi.org/10.1200/JCO. 2010.30.3909.

2. Detmar, S. B., Muller, M. J., Schornagel, J. H., Wever, L. D., \& Aaronson, N. K. (2002). Health-related quality-of-life assessments and patient-physician communication: A randomized controlled trial. Jama, 288(23), 3027-3034. 
3. Velikova, G., Booth, L., Smith, A. B., Brown, P. M., Lynch, P., Brown, J. M., \& Selby, P. J. (2004). Measuring quality of life in routine oncology practice improves communication and patient well-being: A randomized controlled trial. J Clin Oncol, 22(4), 714-724. https://doi.org/10.1200/JCO.2004.06.078.

4. Velikova, G., Keding, A., Harley, C., Cocks, K., Booth, L., Smith, A. B., ... Brown, J. M. (2010). Patients report improvements in continuity of care when quality of life assessments are used routinely in oncology practice: Secondary outcomes of a randomised controlled trial. Eur J Cancer, 46(13), 2381-2388. https://doi.org/10.1016/j.ejca.2010.04.030.

5. Sloan, J. A., Halyard, M., El Naqa, I., \& Mayo, C. (2016). Lessons from largescale collection of patient-reported outcomes: Implications for big data aggregation and analytics. Int J Radiat Oncol Biol Phys, 95(3), 922-929. https://doi.org/10.1016/j.jijrobp.2016.04.002

6. Sloan, J. A., Zhao, X., Novotny, P. J., Wampfler, J., Garces, Y., Clark, M. M., \& Yang, P. (2012). Relationship between deficits in overall quality of life and non-small-cell lung cancer survival. Journal of clinical oncology, 30(13), 14981504. https://doi.org/10.1200/JCO.2010.33.4631

7. Basch, E., Deal, A. M., Dueck, A. C., Scher, H. I., Kris, M. G., Hudis, C., \& Schrag, D. (2017). Overall survival results of a trial assessing patient-reported outcomes for symptom monitoring during routine Cancer treatment. Jama, 318(2), 197-198. https://doi.org/10.1001/jama.2017.7156.

8. Bruera, E. (2008). Routine symptom assessment: Good for practice and good for business. Support Care Cancer, 16(6), 537-538. https://doi.org/10.1007/ s00520-008-0433-1.

9. Health USDo, Human Services FDACfDE, Research, Health USDo, Human Services FDACfBE, Research, Health USDo, Human Services FDACfD, Radiological H (2006). Guidance for industry: Patient-reported outcome measures: Use in medical product development to support labeling claims: Draft guidance. Health Qual Life Outcomes, 4, 79. https://doi.org/10.1186/ 1477-7525-4-79.

10. Aaronson, N. K., \& Snyder, C. (2008). Using patient-reported outcomes in clinical practice: Proceedings of an International Society of Quality of life research conference. Qual Life Res, 17(10), 1295. https://doi.org/10.1007/ s11136-008-9422-6.

11. Niska, J. R., Halyard, M. Y., Tan, A. D., Atherton, P. J., Patel, S. H., \& Sloan, J. A. (2017). Electronic patient-reported outcomes and toxicities during radiotherapy for head-and-neck cancer. Qual Life Res, 26(7), 1721-1731. https://doi.org/10.1007/s11136-017-1528-2.

12. Rathod, S., Gupta, T., Ghosh-Laskar, S., Murthy, V., Budrukkar, A., \& Agarwal, J. (2013). Quality-of-life (QOL) outcomes in patients with head and neck squamous cell carcinoma (HNSCC) treated with intensity-modulated radiation therapy (IMRT) compared to three-dimensional conformal radiotherapy (3D-CRT): Evidence from a prospective randomized study. Oral Oncol, 49(6), 634-642. https://doi.org/10.1016/j.oraloncology.2013.02.013.

13. Rosenthal, D. I., Mendoza, T. R., Fuller, C. D., Hutcheson, K. A., Wang, X. S. Hanna, E. Y., ... Gunn, G. B. (2014). Patterns of symptom burden during radiotherapy or concurrent chemoradiotherapy for head and neck cancer: A prospective analysis using the University of Texas MD Anderson Cancer Center Symptom Inventory-Head and neck module. Cancer, 120(13), 19751984. https://doi.org/10.1002/cncr.28672.

14. Trotti, A., Pajak, T. F., Gwede, C. K., Paulus, R., Cooper, J., Forastiere, A., ... Curran, W. (2007). TAME: Development of a new method for summarising adverse events of cancer treatment by the radiation therapy oncology group. Lancet Oncol, 8(7), 613-624. https://doi.org/10.1016/S14702045(07)70144-4.

15. Huschka, M. M., Mandrekar, S. J., Schaefer, P. L., Jett, J. R., \& Sloan, J. A. (2007). A pooled analysis of quality of life measures and adverse events data in north central cancer treatment group lung cancer clinical trials. Cancer, 109(4), 787-795. https://doi.org/10.1002/cncr.22444.

16. Atherton, P. J., Watkins-Bruner, D. W., Gotay, C., Moinpour, C. M., Satele, D. V. Winter, K. A., ... Sloan, J. A. (2015). The complementary nature of patient-reported outcomes and adverse event reporting in cooperative group oncology clinical trials: A pooled analysis (NCCTG N0591). J Pain Symptom Manag, 50(4), 470-479 e479. https://doi.org/10.1016/j.jpainsymman.2015.04.016.

17. Snyder, C. F., Blackford, A. L., Wolff, A. C., Carducci, M. A., Herman, J. M., Wu, A. W., \& PatientViewpoint Scientific Advisory, B. (2013). Feasibility and value of PatientViewpoint: A web system for patient-reported outcomes assessment in clinical practice. Psychooncology, 22(4), 895-901. https://doi. org/10.1002/pon.3087.

18. Bretscher, M., Rummans, T., Sloan, J., Kaur, J., Bartlett, A., Borkenhagen, L., \& Loprinzi, C. (1999). Quality of life in hospice patients. A pilot study.
Psychosomatics, 40(4), 309-313. https://doi.org/10.1016/S00333182(99)71224-7.

19. Hyland, M. E., \& Sodergren, S. C. (1996). Development of a new type of global quality of life scale, and comparison of performance and preference for 12 global scales. Qual Life Res, 5(5), 469-480. https://doi.org/10.1007/ bf00540019.

20. Cohen, J. (1988). Statistical power analysis for the behavioral sciences, (2nd ed , ). Hillsdale, NJ: Lawerence Earlbaum Associates.

21. Fromme, E. K., Eilers, K. M., Mori, M., Hsieh, Y. C., \& Beer, T. M. (2004). How accurate is clinician reporting of chemotherapy adverse effects? A comparison with patient-reported symptoms from the quality-of-life questionnaire C30. J Clin Ontara, 22(17), 3485-3490. https://doi.org/10.1200/ JCO.2004.03.025

22. Falchook, A. D., Green, R., Knowles, M. E., Amdur, R. J., Mendenhall, W., Hayes, D. N., ... Chera, B. S. (2016). Comparison of patient- and practitionerreported toxic effects associated with Chemoradiotherapy for head and neck Cancer. JAMA Otolaryngol Head Neck Surg, 142(6), 517-523. https://doi. org/10.1001/jamaoto.2016.0656

23. Dueck, A. C., Mendoza, T. R., Mitchell, S. A., Reeve, B. B., Castro, K. M., Rogak, L. J., ... National Cancer Institute PROCSG (2015). Validity and Reliability of the US National Cancer Institute's Patient-Reported Outcomes Version of the Common Terminology Criteria for Adverse Events (PRO-CTCAE). JAMA Oncol, 1(8), 1051-1059. https://doi.org/10.1001/jamaoncol.2015.2639.

\section{Publisher's Note}

Springer Nature remains neutral with regard to jurisdictional claims in published maps and institutional affiliations.

\section{Submit your manuscript to a SpringerOpen ${ }^{\circ}$ journal and benefit from:}

- Convenient online submission

Rigorous peer review

- Open access: articles freely available online

- High visibility within the field

- Retaining the copyright to your article

Submit your next manuscript at $\boldsymbol{\nabla}$ springeropen.com 